Article

\title{
Retrieving Vertical Air Velocity in Convective Cloud over the Tibetan Plateau from TIPEX-U Cloud Radar Doppler Spectra
}

\author{
Jiafeng Zheng', Liping Liu ${ }^{2 *}$, Keyun $\mathrm{Zhu}^{1}$, Jingya Wu ${ }^{3}$, Binyun Wang ${ }^{1}$
}

1. Plateau Atmosphere and Environment Key Laboratory of Sichuan Province, College of Atmospheric Sciences, Chengdu University of Information Technology, Chengdu, 610225, China; zjf@cuit.edu.cn (J. Z.); zhuky@cuit.edu.cn (K. Z.); bywang@cuit.edu.cn (B. W.)

2. State Key Lab of Severe Weather, Chinese Academy of Meteorological Science, Beijing, 100081, China; lpliu@camscma.cn (L.L.)

3. Institute of Atmospheric Physics, Chinese Academy of Sciences, Beijing, 100029, China; 653724930@qq.com (J.W.)

\begin{abstract}
In the summertime, convections occur frequently over the Tibetan Plateau (TP) because of the large dynamic and thermal effects of the landmass. Measurements of vertical air velocity in convective cloud are useful for advancing our understanding of the dynamic and microphysical mechanisms of clouds and can be used to improve the parameterization of current numerical models. This paper presents a technique for retrieving high-resolution vertical air velocity from convective cloud over the TP, by using Doppler spectra from a vertically pointing Ka-band cloud radar. The method is based on the development of a "smallparticle-traced" idea and the necessary data processing and uses three modes of radar. Spectral broadening corrections, uncertainty estimations, and result merging are used to ensure accurate results. Qualitative analysis of two typical convective cases shows that the retrievals are reliable and agree with the expectant results inferred from other radar measurements. A quantitative retrieval of vertical air motion from a ground-based optical disdrometer is used to preliminarily validate our radar-derived results. The comparison illustrates that while the data trends from the two methods of retrieval are similar, with the updrafts and downdrafts coinciding, cloud radar has a much higher resolution and can reveal the small-scale variation of vertical air motion.
\end{abstract}

Keywords: vertical air velocity; millimeter-wave cloud radar; convective cloud; Tibetan Plateau

\section{Introduction}

The Tibetan Plateau (abbreviated to TP) is the largest and highest plateau on our planet, with an average altitude of over 4000 meters and complicated terrain. Because of the great dynamic and thermal effects of its landmass, TP can significantly impact the circulation, climate, and weather of East Asia and the entire Northern Hemisphere [1-5]. In the summertime, vertical air motions over TP are stronger than over other areas because of significant ground heating, leading to frequent convections. Several early investigations suggested that convective cloud over TP accounts for approximately $60 \%$ of the total cloud,

\footnotetext{
" Corresponding author: Liping Liu, Email: 1pliu@camscma. cn. Address: N0.46, Zhongguancun
} South Street, Haidian District, Beijing ,China. 
with convection occurrence rates 1.5 times higher than over other regions of China. Indeed, there may be up to 300 convection cells on the plateau per day [6-7]. Under favorable synoptic conditions, these convections can move eastwards to central and eastern China and cause bad weather [8-9]. Moreover, the specific natural environment on TP results in unique cloud and precipitation properties, when compared to other low-altitude regions [10-11], and can prominently limit the simulation capabilities of current numerical models. As a kinetic factor, vertical air motions play an important role during the formation and evolution of convective cloud processes. However, accurate and high-resolution measurements of vertical air velocity by remote sensing equipment are lacking. These types of data would help us to better understand the dynamical processes of clouds during their lifetime and promote the parameterization of physical mechanisms within models at many scales.

Millimeter-wave Doppler radars (generally termed "cloud radar") have been extensively developed over the last 20 years, becoming a major tool for the observation of cloud and lightprecipitation because of their high sensitivity and resolution. Cloud radars operate mainly in the Ka-band ( $\sim 35 \mathrm{GHz}$, with a wavelength of $8 \mathrm{~mm})$ and W-band $(\sim 94 \mathrm{GHz}$, with a wavelength of $3 \mathrm{~mm}$ ), and their excellent performance in cloud detection has been demonstrated by many studies [12-16]. As an antenna is vertically pointed, cloud radar can measure the vertical motions of cloud particles in the atmosphere. However, the returned Doppler velocities consist of both particle terminal velocities and air motions [17]. Moreover, for convective clouds, radarmeasured Doppler velocities can be influenced by small-scale turbulence and wind shear within the sampling volume [18]. Thus, the deviation of vertical air velocity in convective cloud from oriented cloud radar measurements is difficult.

In several early studies, meteorologists tried to establish an explicit relationship between radar reflectivity $(\mathrm{Z})$ and mean fall velocity of particles $\left(\mathrm{V}_{\mathrm{t}}\right)$ in quiet air, because both $\mathrm{Z}$ and $\mathrm{V}_{t}$ are proportional to hydrometeor diameter (D). The relationship was produced by theoretical derivations and practical observations [19-22]. Using this relationship, vertical air velocity can be deduced by subtracting $V_{t}$ estimated by radar-measured $\mathrm{Z}$ from the radar-measured mean Doppler velocity $(\overline{\mathrm{V}})$. However, the prerequisites of this method are knowledge of particle size distribution and an accurate $\mathrm{V}_{\mathrm{t}}-\mathrm{D}$ equation, which are complicated and currently undefined for convective ice cloud. For a radar received signal, the Fast Fourier Transform, or other spectrum analysis algorithms, can be utilized to obtain the cloud radar Doppler spectra, which contain a wealth of information on cloud properties, vertical air motions, and turbulence. Under specific sets of conditions, cloud radar spectra provide more reliable source data for the retrieval of vertical air velocity. For the condition of strong precipitation, W-band cloud radar will suffer from Mie scattering due to its very short wavelength, and the signature of the observed spectra (modulated by the backscattering function that in the Mie regime oscillates between fixed maxima and minima) can be used as reference points to retrieve the air velocity. This special technology was first mentioned by Lhermitte and has been verified and applied by Kollias to derive the updrafts and downdrafts in strong convective precipitation [18, 23]. However, despite its attraction, this novel usage is not applicable to Ka-band millimeter-wave radar and does not work under conditions of no precipitation or light precipitation. In the presence of small particles, such as liquid droplets and small ice crystals, within the cloud radar sampling volume, vertical air velocity can be directly estimated by using the velocity bin of 
these small targets, regarded as tracers of clear-air motions in the measured spectra [24-27]. This technology may be widely applied to different cloud types, but spectral broadening caused by turbulence and wind shear must be considered as unnegligible uncertainties. Due to the rapid change and complicated dynamic structure of convective cloud, the retrieval of vertical air velocity is still a challenge. Moreover, convections over TP have certain characteristics, such as ice phases, weaker intensities, smaller scales, and shorter life cycles, which will worsen the retrieval difficulty.

In this manuscript, a technique for retrieving vertical air velocity in convective cloud over TP is introduced. This technique is designed for a Ka-band solid-state cloud radar used in the Third Tibetan Plateau Meteorological Science Experiment. In the following section, details of the cloud radar and experiment are described. Section 3 depicts the data processing of radar recorded Doppler spectra. Section 4 details an integrated retrieval technique using three-mode Doppler spectra. Section 5 analyzes the retrieval results of two cases and validates the reliability of the presented technique.

\section{Experiment, Instrument, and Measurements}

In summer 2013, the Chinese Academy of Meteorological Sciences (CAMS) and several other research institutes launched the 3rd Tibetan Plateau Meteorological Science Experiment (TIPEX-III). TIPEX-III is the newest and largest meteorological experiment on the TP, with a duration of ten years. One purpose of this experiment is to use multi-wavelength radars, microwave radiometers, disdrometers, and other ground-based equipment to construct a three-dimensional observation system for cloud and precipitation. The first-stage mission of this experiment was achieved in the summers of 2014 and 2015 [28].

The primary instrument is a vertically pointing millimeter-wave cloud radar deployed at Naqu, of the Tibet autonomous region (latitude: $32^{\circ} 29^{\prime} \mathrm{N}$; longitude: $92^{\circ} 04^{\prime} \mathrm{E}$; altitude: $4,507 \mathrm{~m}$ ), to detect vertical profiles of cloud and weak precipitation. This radar was developed by CAMS and the 23rd Institute of China Aerospace Science and Technology Corporation. To better adapt to the hostile environment of the plateau and to ensure a long-term operating capacity, the radar uses a solid-state transmitter and is equipped with an independent container. The radar operates at $33.44 \mathrm{GHz}$ (a wavelength of $8.9 \mathrm{~mm}$ ) with a peak power of over $100 \mathrm{~W}$. The primary aim of using the Ka-band is to obtain as much backscattered energy as possible under the Rayleigh scattering mechanism, while reducing the attenuation effects of air and precipitation particles. A 2-m diameter Cassegrain antenna is used to achieve a $53 \mathrm{~dB}$ gain and to form a 0.3degree beamwidth, so that the radar horizontal resolution is only $26 \mathrm{~m}$ at $5 \mathrm{~km}$ (AGL). The transmitted pulse width is $0.2 \mu \mathrm{s}$, corresponding to a vertical resolution of $30 \mathrm{~m}$. Thus, the radar can provide fine-resolution mapping of cloud structure and boundaries. The radar measurements include the initial Doppler spectrum, reflectivity, mean Doppler velocity, spectrum width, linear depolarization ratio, skewness, and kurtosis. The major technical and operational parameters of the radar are summarized in Table 1. 
Table 1. Major technical and operational parameters of TIPEX-III cloud radar

\begin{tabular}{ll}
\hline \multicolumn{1}{c}{ Items } & \multicolumn{1}{c}{ Technical specifications } \\
\hline Radar system & Doppler, solid-state, depolarization, multi-mode \\
Frequency & $33.44 \mathrm{GHz}$ \\
Wavelength & $8.9 \mathrm{~mm}$ \\
Transmitted peak power & $\geq 100 \mathrm{~W}$ \\
Sensitivity & $-38 \mathrm{dBZ} @ 5 \mathrm{~km}$ \\
Antenna diameter & $2 \mathrm{~m}$ \\
Antenna gain & $\geq 53 \mathrm{~dB}$ \\
Pulse width & $0.2 \mu \mathrm{s} 、 12 \mu \mathrm{s}$ \\
Beam width & $0.3^{\circ}$ \\
PRF & $8333 \mathrm{~Hz}$ \\
Range gate number & 510 \\
& Height: $120 \mathrm{~m} \sim 15.3 \mathrm{~km}$ \\
Detection range & Measurable reflectivity range: $-50 \sim 30 \mathrm{dBZ}$ \\
& Unambiguous velocity range: $-18.54 \sim+18.54 \mathrm{~m} / \mathrm{s}($ maximum $)$ \\
Resolution & Temporal resolution: $\sim 9 \mathrm{~s}($ adjustable) \\
& Vertical resolution: $30 \mathrm{~m}$ \\
& horizontal resolution: $26 \mathrm{~m} @ 5 \mathrm{~km}$ \\
& Original data: Doppler spectra \\
Measurements & Spectral moments: reflectivity $(\mathrm{Z})$, mean Doppler velocity $(\overline{\mathrm{V}})$, \\
& spectrum width $\left(\sigma_{\mathrm{v}}\right)$, linear depolarization ratio $(\mathrm{LDR}), \mathrm{skewness}$ \\
& $\left(\mathrm{S}_{\mathrm{k}}\right)$, kurtosis $\left(\mathrm{K}_{\mathrm{t}}\right)$ and etc. \\
\hline
\end{tabular}

For the radar system, the key performance indicators, such as its sensitivity, detectable range, unambiguous velocity, and velocity resolution, are a compromise. So, to meet the requirements for cloud and precipitation observation over the TP at different heights and with different intensities, three operational modes were designed for this radar: the boundary mode $(\mathrm{BL})$, the cirrus mode (CI), and the precipitation mode (PR). The three modes were configured with different pulse widths and coherent and incoherent integration numbers. BL and PR are used mainly to observe cloud and light precipitation in the low and middle levels. Short pulses ensure a small blind range for both modes, while the minimum detectable reflectivity is relatively large. The major difference between $\mathrm{BL}$ and $\mathrm{PR}$ is that four times more coherent integration is performed for $\mathrm{BL}$, which reduces the minimum detectable reflectivity by $6 \mathrm{~dB}$, decreases the Nyquist velocity by a factor of 3 , and increases the velocity resolution by a factor of 3. CI is used for cloud detection in the middle and upper levels, where clouds are characterized by high altitude and small or moderate reflectivity and vertical motion. For this purpose, pulse compression (with a wide pulse of $12 \mu \mathrm{s}$ ) and twice as much coherent integration were used to decrease the minimum detectable reflectivity by $20 \mathrm{~dB}$. CI has a high detection range and moderate Nyquist velocity and velocity resolution, but a larger blind range from $0.12 \mathrm{~m}$ to $2.01 \mathrm{~km}$. The radar system operates the three modes periodically; that is, once the radial measurement is finished in one mode, the radar immediately switches to another. Each mode will cause $\sim 3 \mathrm{~s}$ for signal dwell and data processing, so the final temporal resolution of the radar is $\sim 9 \mathrm{~s}$. The observations collected by the three modes are saved separately and a merging algorithm is used to produce the integrated spectral moments [28]. Operational parameters of the three modes are summarized in Table 2. 
Table 2. Operational parameters of three modes.

\begin{tabular}{lccc}
\hline \multicolumn{1}{c}{ Parameters } & BL & Mode & PR \\
\hline Detectable range $(\mathrm{km})$ & $0.12-7.5$ & $2.04-15.3$ & $0.12-12$ \\
Pulse width $(\mu \mathrm{s})$ & 0.2 & 12 & 0.2 \\
Pulse period $(\mu \mathrm{s})$ & 120 & 120 & 120 \\
Pulse repetition frequency $(\mathrm{Hz})$ & 8333 & 8333 & 8333 \\
Number of coherent integration & 4 & 2 & 1 \\
Number of incoherent integration & 16 & 32 & 64 \\
Sensitivity & $-24 \mathrm{dBZ} @ 5 \mathrm{~km}$ & $-38 \mathrm{dBZ} @ 5 \mathrm{~km}$ & $-18 \mathrm{dBZ} @ 5 \mathrm{~km}$ \\
Spectral bin number & 256 & 256 & 256 \\
Nyquist velocity $(\mathrm{m} \mathrm{s}-1)$ & \pm 4.635 & \pm 9.27 & \pm 18.54 \\
velocity resolution $(\mathrm{cm} \mathrm{s}-1)$ & 3.64 & 7.27 & 14.54 \\
\hline
\end{tabular}

To simply assess the detection capability of this radar, the probability distribution of measured reflectivity at different heights, collected from June to August 2014 and July to September 2015, was calculated. As shown in Figure 1, the realistic minimum detectable reflectivities of $\mathrm{BL}$ and $\mathrm{CI}$ at different heights are coincident with the theoretical results (marked curves), indicating the expected capability for weak cloud observation. CI has a $20 \mathrm{~dB}$ and a 14 $\mathrm{dB}$ weaker minimum detectable reflectivity than $\mathrm{PR}$ and $\mathrm{BL}$, respectively. However, strong reflectivity, greater than $30 \mathrm{dBZ}$, was hardly observed due to signal attenuation and Mie scattering at this wavelength. Zheng investigated the measurement deviation of this radar by comparing it with a C-band vertically pointing radar and suggested that a $2 \mathrm{~dB}$ bias may exist under light precipitating conditions, with maximum reflectivity of no more than $27 \mathrm{dBZ}$ [29]. Figure 2 presents an example of $3 \mathrm{~h}$ time-height cross sections of reflectivity from the three different modes made on July 20, 2015. It indicates that CI can detect much weaker reflectivity in the cloud boundary than BL and PR can, at heights above $2.01 \mathrm{~km}$. Some artifacts are caused by the pulse compression on the cloud top or under the cloud base. Two layers of cirrus can be clearly found in CI mapping but are obscured in the BL and PR mappings. BL is more appropriate for weak cloud observation (see the small-scale cumulus near $1 \mathrm{~km}$ ) below 2.01 $\mathrm{km}$, when compared with PR. In contrast, PR is more suitable than BL for light precipitation observation. 


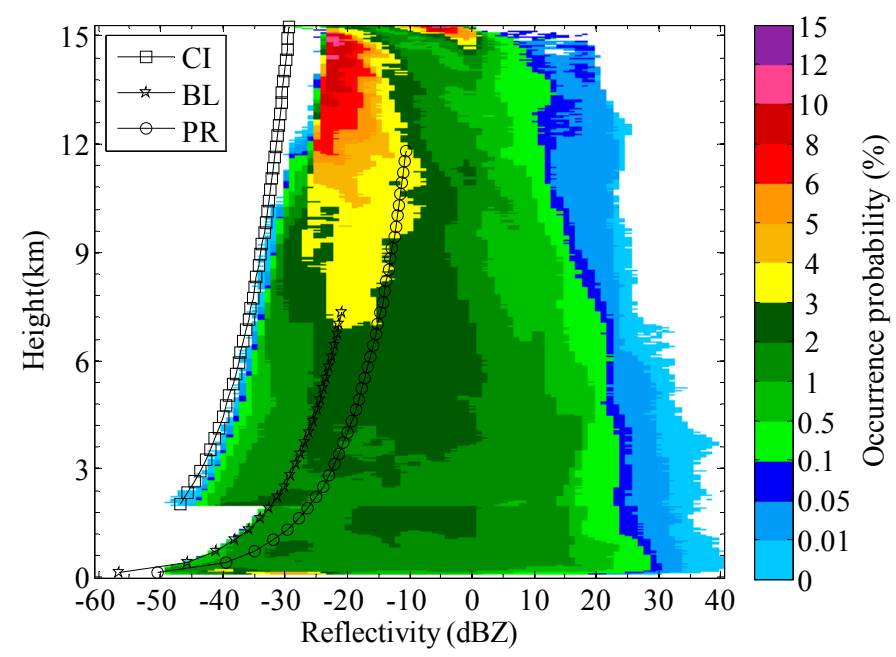

Figure 1. Probability distribution of radar-measured reflectivity at different altitude collected from June to August 2014 and July to September 2015 on the TP, the marked curves represent the theoretical sensitivity of three operational modes.

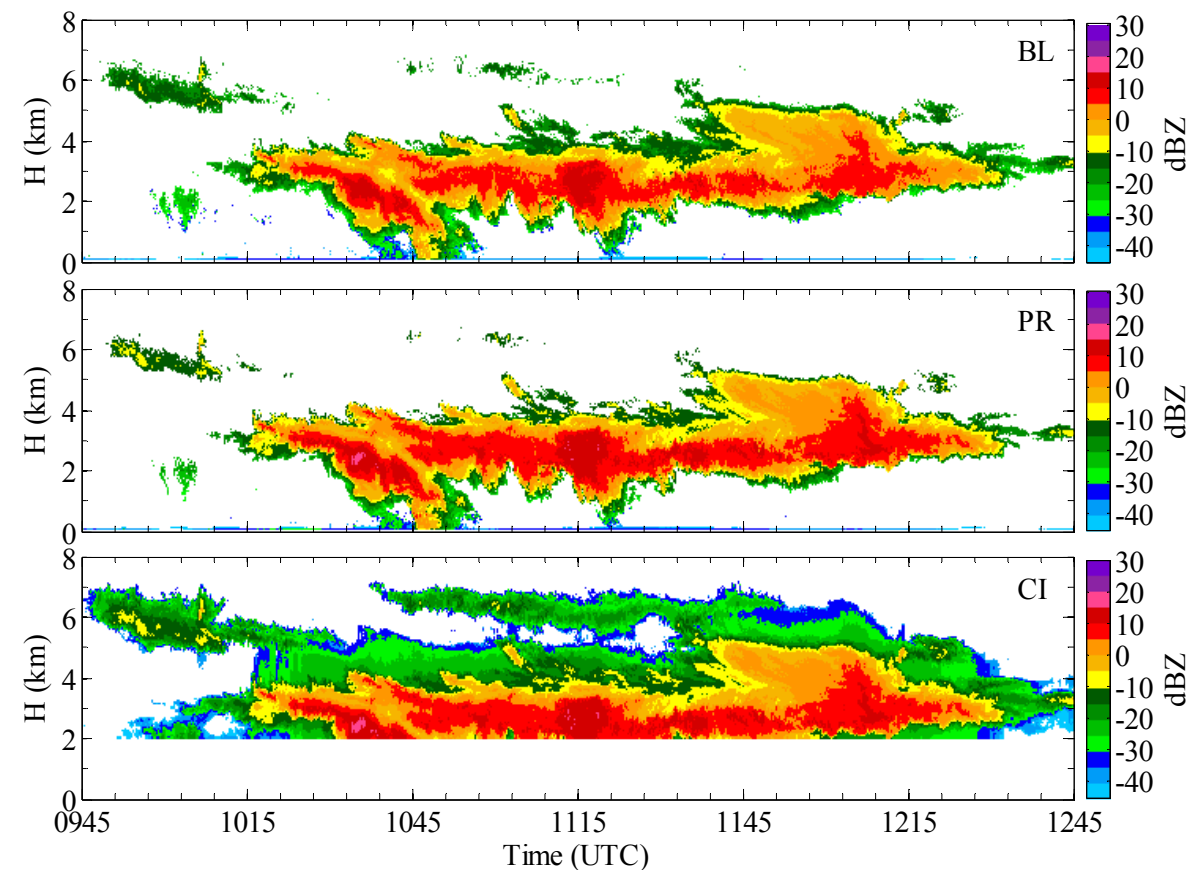

Figure 2. An example of $3 \mathrm{~h}$ time-height cross-sections of reflectivity from three operational modes observed on 20 July 2015.

\section{Data Processing of Cloud Radar Doppler Spectra}

The TIPEX-III cloud radar produces vertically resolved measurements of Doppler spectra, which consist of 256 spectral bins and represent the distribution of returned radar power as a function of hydrometer radial velocity in the sampling volume. Cloud radar Doppler spectra have been commonly used for cloud property retrieval and microphysical studies because of their wealth of information on drop size distribution, turbulence, vertical air motion, and so 
on. However, Doppler spectra simultaneously contain radar noise and meteorological signals and suffer from velocity aliasing under special conditions. So, before further application, sophisticated data processing procedures are presented here.

Firstly, a 3-point boxcar averaging window was used to smooth all Doppler spectra to improve spectral clarity and decrease the fluctuation of radar noise. Noise level calculation was a crucial step to accurately separate the radar noise and cloud signal. An objective method presented by Hildebrand and Sekhon has been commonly used for millimeter-wave cloud radar studies [30-31, 18, 26]. However, a recent study argues that this approach can overestimate the radar noise power and so is not appropriate for solid-state cloud radar. In contrast, a segmental approach reported by Monique can achieve better accuracy and stability $[29,32]$. So, in this paper, a simple 8-segment technology was utilized to calculate radar noise level, i. e., orderly dividing the spectra into eight segments, with the lowest integrated power segment regarded as being dominated by radar noise, without any meteorological signal. The mean power of this segment was calculated as the noise level for the whole Doppler spectrum. Subsequently, all continuous spectral bins above noise level were picked and further judged by a SNR (Signal-to-Noise Ratio) threshold and a bin-number threshold, since cloud signal typically has a stronger power and larger spectral width than noise. Only the consecutive signals with the first three powers were reserved, and several spectral parameters, such as SNRs, left endpoints, right endpoints, and peaks, were also recorded.

Due to more coherent integration numbers being used, radar BL and CI modes obtained relatively small measurable velocity ranges from -4.635 to $4.635 \mathrm{~m} \mathrm{~s}^{-1}$ and from -9.27 to $9.27 \mathrm{~m}$ $\mathrm{s}^{-1}$, respectively. In the presence of large droplets or strong airflows in convective systems, the measured Doppler spectra may be folded, which leads not only to discontinuity of the cloud signal, but also to spectral moments (such as mean Doppler velocity and spectrum width) generating significant errors. Here, a previously presented algorithm was used for velocity dealiasing of Doppler spectra [29]. This algorithm can iteratively judge and correct the folded Doppler spectra from cloud top to base and can automatically determine the folding type, a property that is caused by either hydrometer rapid sinking or strong updraft in the interior of the convective system. After the ambiguous Doppler spectra are corrected, the corresponding spectral parameters were also readjusted. BL and CI Doppler spectra, collected from TIPEX-III in the summers of 2014 and 2015, were used for assessing the dealiasing effects, by comparison with the corresponding spectra of the PR mode. Results show a success rate of $99.8 \%$ of this algorithm when used in only a one-time fold. Figure 3 shows a typical case of a convective cloud, collected at 10:00 UTC on July 5, 2014, by CI mode. From the mapping of the original spectra (Figure 3(a)), parts of the ambiguous signal can be found near the $6 \mathrm{~km}$, due to strong updraft. Parts of the ambiguous signal also exist below $4.3 \mathrm{~km}$, because of rapid fall speeds of ice crystals and raindrops. After dealiasing (Figure 3(b)), these two ambiguous signal parts were identified and corrected, so that the upper one is shifted to the left terminal and the lower one is shifted to the right terminal. Two profiles of the mean Doppler velocity were calculated from the original and the corrected spectra (Figure 3(c)), showing one maximum deviation approaching $4 \mathrm{~m} \mathrm{~s}^{-1}$ at $6 \mathrm{~km}$ and another of $-6 \mathrm{~m} \mathrm{~s}^{-1}$ at $3.4 \mathrm{~km}$. Meanwhile, two profiles of the estimated spectrum widths presented more serious deviations at the spectral folded regions, with a maximum bias of $4.5 \mathrm{~m} \mathrm{~s}^{-1}$ at $6 \mathrm{~km}$ and another maximum bias of $7.5 \mathrm{~m} \mathrm{~s}^{-1}$ at $3.4 \mathrm{~km}$. 

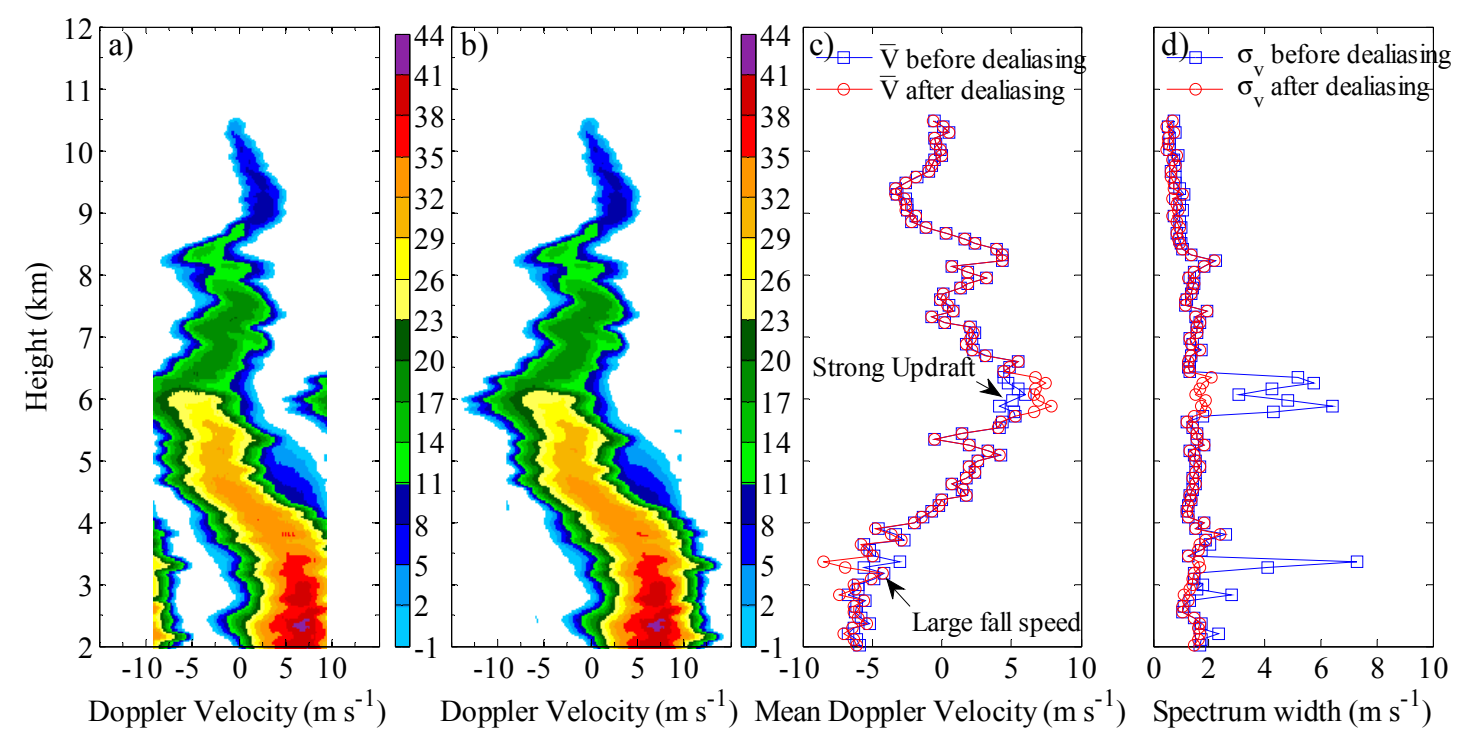

Figure 3. A velocity dealiasing case made from a convective cloud collected at 10:00 UTC on 5 July 2014 by CI mode; a) is the original Doppler spectra at different height; b) is the corresponding corrected spectra after dealiasing; c) is the estimated mean Doppler velocity by a) and b); d) is the estimated spectrum width by a) and $b$ ).

\section{Retrieval Method}

\subsection{Essential Idea}

Doppler velocities of cloud signal in the resolved spectra are typically proportional to the hydrometeor diameters, based on the assumption that they experience uniform vertical air motion and homogeneous turbulence in the radar sampling volume. Thus, the left spectral edge of an identified cloud signal should be generated by the smallest cloud particles. Because of the limited size of these small targets, their corresponding fall speed will be negligible when compared with the active vertical air motions in convective cloud on the TP, which are one/two orders of magnitude larger. So, we can directly derive the vertical air velocity by using the left spectral bin, which regards the smallest cloud particles as a tracer of clear-air motions. This simple idea (here referred to as "small-particle-traced idea") was previously described by Gossard and Kollias and then further validated by Shupe [24-26]. Figure 4 shows a schematic of the small-particle-traced idea, where the $\omega_{\text {initial }}$ represents the initial estimate of vertical air velocity. 


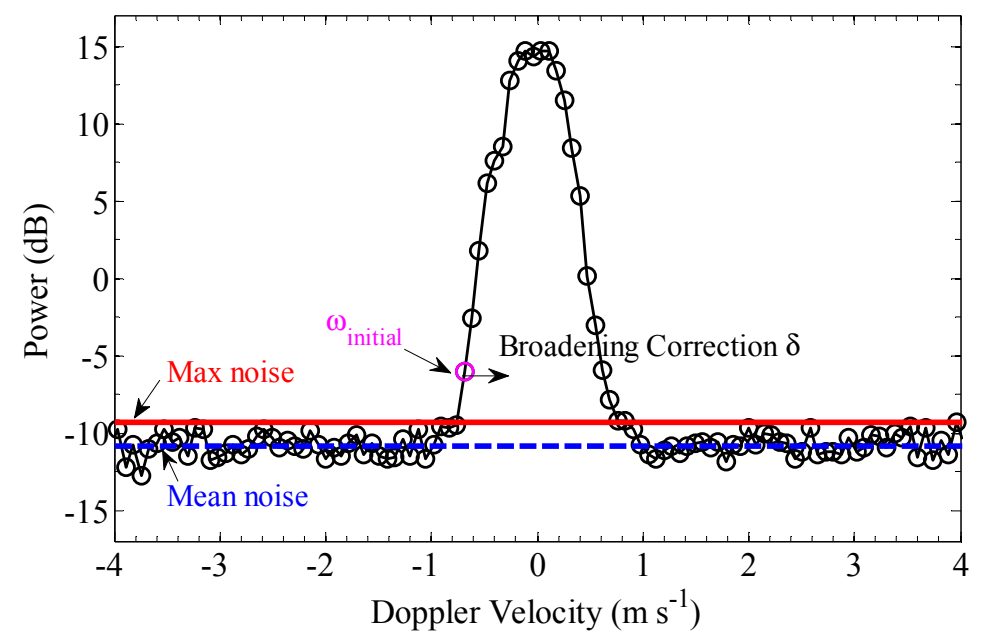

Figure 4. A schematic of the small-particle-traced idea, the blue dashed line is the estimated radar mean noise, the solid red line is the max noise, the $\omega_{\text {initial }}$ represents the initial retrieval of vertical air velocity.

\subsection{Integrated Retrieval Technique Using Multimode Doppler Spectra}

In the use of Doppler spectra from TIPEX-III cloud radar for retrieving vertical air motions in convective cloud, there are three aspects that require further consideration:

(1) The three operational modes have different sensitivities, detectable height ranges, Nyquist velocities, and spectral velocity resolutions. These key performance parameters are crucial for the estimation of vertical air velocity. Thus, comprehensive usage of the multimode data needs to be considered to obtain more reasonable and accurate results.

(2) Convective cloud over TP commonly varies rapidly with small scales, and thus the measured radar Doppler spectra in a sampling volume can be broadened by the active turbulence, wind shear, and inhomogeneous horizontal wind, which will cause bias to the retrieval results. Therefore, spectral broadening needs to be further revised.

(3) Because of a low melting layer altitude of approximately $1 \mathrm{~km}$ on TP, hydrometeors in the interior of convections exist mostly in the ice phase, which typically involves greater diameters than the liquid phase. Furthermore, the rain droplets under the cloud base also have relatively large diameters. Therefore, as the fall velocities of the traced targets in the radar sampling volume cannot be neglected, the possible biases of results from the small-particle-traced idea need to be documented.

Based on these three considerations, an integrated technique using multimode Doppler spectra is proposed for comprehensive retrieval of vertical air velocity. Figure 5 shows a flow diagram of the method. For the height range $2.01-15.3 \mathrm{~km}$, Doppler spectra from CI are used, because this mode is more sensitive at detecting smaller cloud targets and has a higher detectable range. For the height range below $2.01 \mathrm{~km}(0.12-1.98 \mathrm{~km})$, the reflectivity is utilized to judge whether BL or PR would be more appropriate for the retrieval. If the reflectivity is greater than $-10 \mathrm{dBZ}$ or the echo base has approached to the near-ground, then Doppler spectra of PR are selected, because the measurements correspond to a relatively strong or precipitating 
cloud and PR has a wider velocity range and a robust dynamic range. On the contrary, if the reflectivity is lower than $-10 \mathrm{dBZ}$ and the echo base is above the near-ground, then the measurements correspond to weak cloud, and Doppler spectra of BL are chosen because of their higher velocity resolution and better sensitivity. After data selection, vertical air velocity can be derived from the three modes by the small-particle-traced idea and its necessary data processing.

Under actual conditions, radar-measured spectra are broader than expected from the cloud particle size distribution alone because of the temporal and spatial filters that introduce additional broadening. The Doppler spectrum variance for a vertically pointing radar can be written as [27]

$$
\sigma_{D}^{2}=\sigma_{D S D}^{2}+\sigma_{T}^{2}+\sigma_{S}^{2}+\sigma_{B}^{2}
$$

where $\sigma_{D S D}^{2}$ is the variance induced by the particle size distribution, $\sigma_{T}^{2}$ is the variance caused by turbulence, $\sigma_{S}^{2}$ is the variance due to wind shear, and $\sigma_{B}^{2}$ is the variance introduced by a finite radar beamwidth. For retrieval of vertical air velocity from an observed Doppler spectrum, without the knowledge of $\sigma_{D S D}^{2}$, the latter three terms are used to correct for the effects of non-hydrometeor size distribution broadening of the left edge of the spectrum.

For a symmetric Gaussian antenna, $\sigma_{B}^{2}$ can be estimated by using the horizontal wind speed, $U$ ( $\mathrm{m} \mathrm{s}^{-1}$, obtained by radiosonde), and the radar beamwidth $(\theta)$ [17] as follows:

$$
\sigma_{B}^{2}=\frac{U^{2} \theta^{2}}{2.76} \text {. }
$$

$\sigma_{S}^{2}$ is caused by vertical $\left(k_{h}\right)$ and horizontal $\left(k_{v}\right)$ shear in the vertical winds (both in $s^{-1}$ ), and this term can be estimated from equation (3) [17, 25-26]. The first term represents transverse shear and the second term represents radial shear. $R$ is the distance from target to radar, $\Delta$ is the radar gate length, and both $k_{h}$ and $k_{v}$ can be calculated by the mean Doppler velocity across three adjacent points.

$$
\sigma_{S}^{2}=\frac{k_{h}^{2} R^{2} \theta^{2}}{2.76}+\frac{k_{v}^{2} \Delta^{2}}{12}
$$

Turbulent broadening of the spectrum variance can be derived by using the temporal variance of the measured mean Doppler velocity, $\sigma_{v m}^{2}$, with the assumption that the length scales of the turbulent eddies observed by the radar are within the inertial subrange of the turbulence spectrum [33-34]. O'Connor et al. (2005) showed that the relationship between $\sigma_{T}^{2}$ and $\sigma_{v m}^{2}$ can be described by a simple form:

$$
\frac{\sigma_{T}^{2}}{\sigma_{v m}^{2}}=\frac{L_{s}^{2 / 3}}{L_{l}^{2 / 3}-L_{s}^{2 / 3}} .
$$

In equation (4), $L_{s}$ is the horizontal length of the radar sampling volume for a $2 \mathrm{~s}$ dwell time and $L_{l}$ represents the larger eddies passing through the effective sampling volume that results from an average of the radar observations over $60 \mathrm{~s}$ [27]. $L_{s}$ and $L_{l}$ can both be given as $L=$ $U t+2 R \sin (\theta / 2)$, where $t$ is the obervation time.

After estimating these three spectral broadening components, a correction factor $\delta(\mathrm{m}$ $\mathrm{s}^{-1}$ ) can be calculated, by which the initial air velocity $\omega_{\text {initial }}$ is increased in the direction of the arrow in Figure $4 . \delta$ can be written as 


$$
\delta=\sigma_{D}-\sqrt{\sigma_{D}^{2}-\left(\sigma_{T}^{2}+\sigma_{S}^{2}+\sigma_{B}^{2}\right)} .
$$

For relatively strong convective clouds or light precipitation, the smallest traced targets in the radar sampling volume may possibly have nonnegligible sizes, corresponding to terminal speeds that affect the accuracy of the derived air velocities. However, these retrieval biases are difficult to estimate because the particles in convective clouds on the TP have unresolved phases or habits. So, to summarize, $Z_{\text {traced }}$ (in dBZ) is calculated, which can represent the intensity of the traced targets. $Z_{\text {traced }}$ can document a qualitative reliability and bias for the retrieval; a smaller value of $Z_{\text {traced }}$ represents a more reliable and accurate result, and conversely a larger value of $Z_{\text {traced }}$ can indicate a larger retrieved bias.

After this, a simple procedure is performed, based on the adjacent deviation between 2.01 $\mathrm{km}$ and $1.98 \mathrm{~km}$, to shift the results below $2.01 \mathrm{~km}$, that is, using the retrieval profile of the upper range with a better accuracy because of the high sensitivity required to amend the retrieval profile of the lower range. Finally, the three kinds of results can be merged to form a complete profile of vertical air velocity.

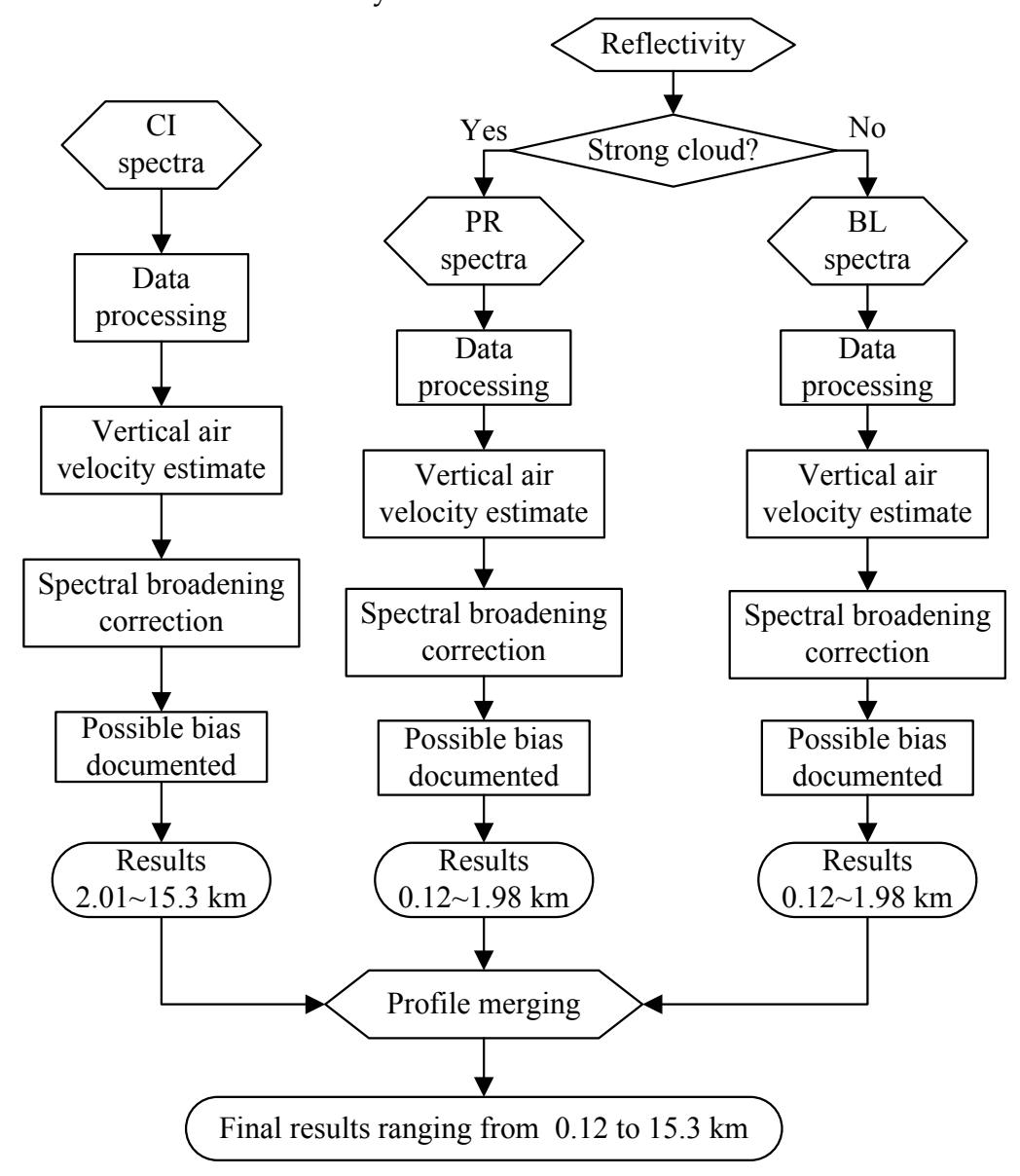

Figure 5. A flow diagram for the integrated technique for retrieval of vertical air velocity using multi-mode Doppler spectra.

\section{Result Analysis and Validation}

\subsection{Two Typical Cases}


Various types of convective clouds frequently occur in the summertime on the TP. Under different synoptic conditions, the clouds may have different macro and micro properties. The two typical cases selected below are used to study the characteristics of cloud processes and vertical air motions.

The first case is a series of fair-weather shallow cumuli collected from 19:00 to 21:30 UTC on July 16, 2015, during a typical convection caused by a weak unstable aerosphere from postmeridian ground heating of the plateau, with no synoptic system. The $2.5 \mathrm{~h}$ cloud radar measurements and retrievals are shown in Figure 6. From the mapping, a series of small-scale cumuli are found at $2-3 \mathrm{~km}$ with a reflectivity range from -30 to $5 \mathrm{dBZ}$. An alternative variation of radar-measured reflectivity $(Z)$, mean Doppler velocity (MV), and spectrum width $\left(\sigma_{v}\right)$ across the cumuli, indicating that inhomogeneous vertical air motions $(\omega)$ and microscopic properties have been generated in the interior of the clouds, a feature that is strikingly similar to the aircraft observations from previous studies [35]. As expected, the derived vertical air velocities show updrafts that occur in the middle of cumulous cells with the cores corresponding to positive MV and downdrafts located on both sides. Figure 7 shows the curves of the height-averaged results of reflectivity, spectrum width, and vertical air velocity. It indicates that the general trends of air velocity and reflectivity are similar; that is, the updrafts are associated with high cloud radar reflectivities, and downdrafts are correlated with low reflectivities. The cloud-inner updrafts can be produced by both environmental upflow and potential heating, with an average speed of $1 \mathrm{~m} \mathrm{~s}^{-1}$, and the downdrafts can be caused by mixing of the environmental air at cloud top and evaporative cooling, with an average speed of $-0.3 \mathrm{~m}$ $\mathrm{s}^{-1}$. Lower spectrum widths less than $0.5 \mathrm{~m} \mathrm{~s}^{-1}$ are observed in the updraft areas, suggesting less turbulent flow associated with relatively weak horizontal variability of vertical air motions and a gradual vertically accelerating motion. However, at the edges of the updraft, the spectral widths increase to about $0.5-1 \mathrm{~m} \mathrm{~s}^{-1}$, particularly in the narrow areas between the updrafts and downdrafts. This broadening of the spectra may be due to sharp horizontal gradients in the vertical wind. The intensity of the smallest traced targets $\left(Z_{\text {traced }}\right)$ is low with a range from -48 to $-33 \mathrm{dBZ}$, demonstrating that the retrieval results can be quite accurate, with a negligible terminal falling speed when compared with the air motions. 


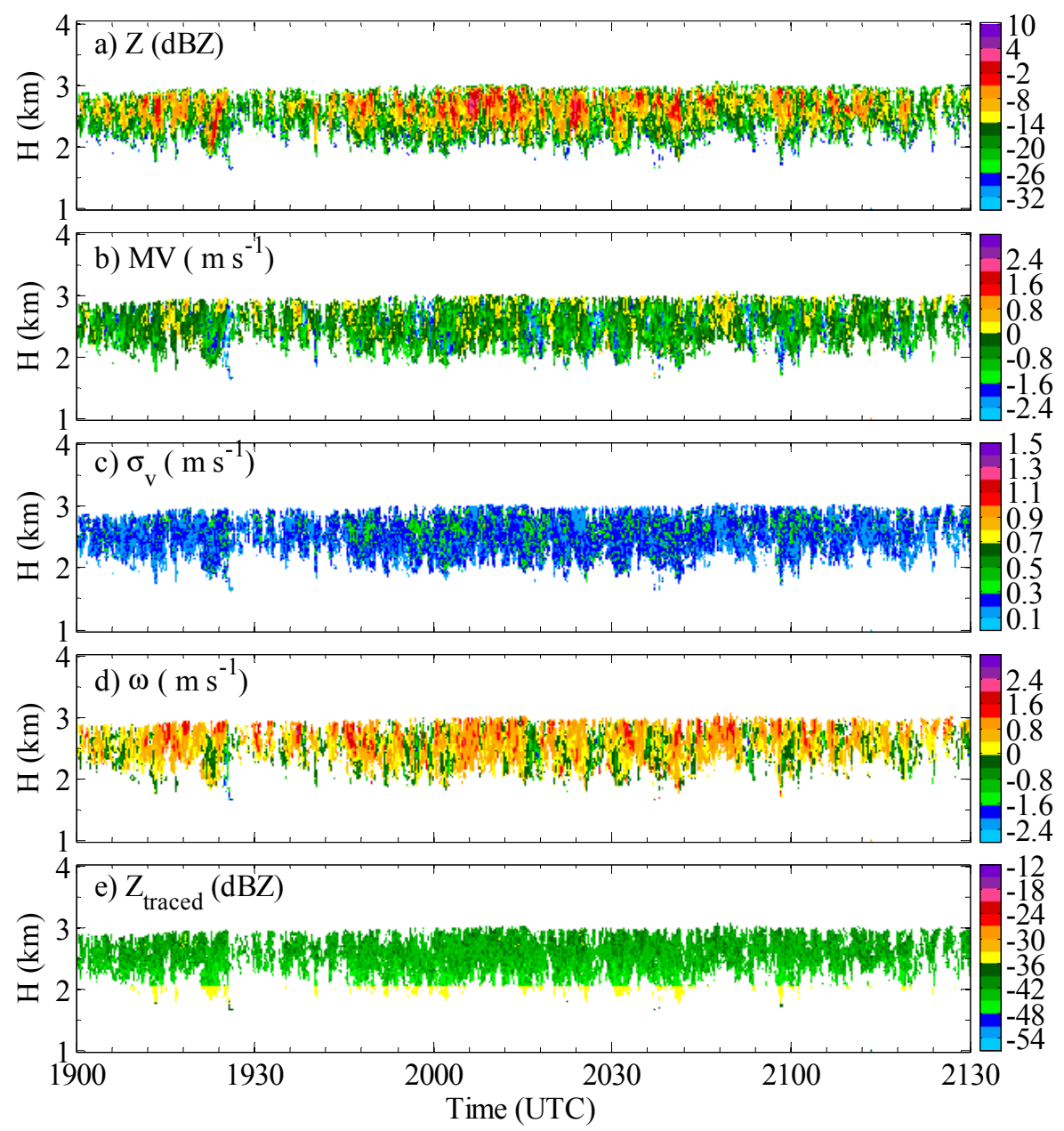

Figure 6. Cloud radar measurements and retrieval results of a series of fair-weather shallow cumuli collected from 19:00 to 21:30 UTC on 16 July 2015.
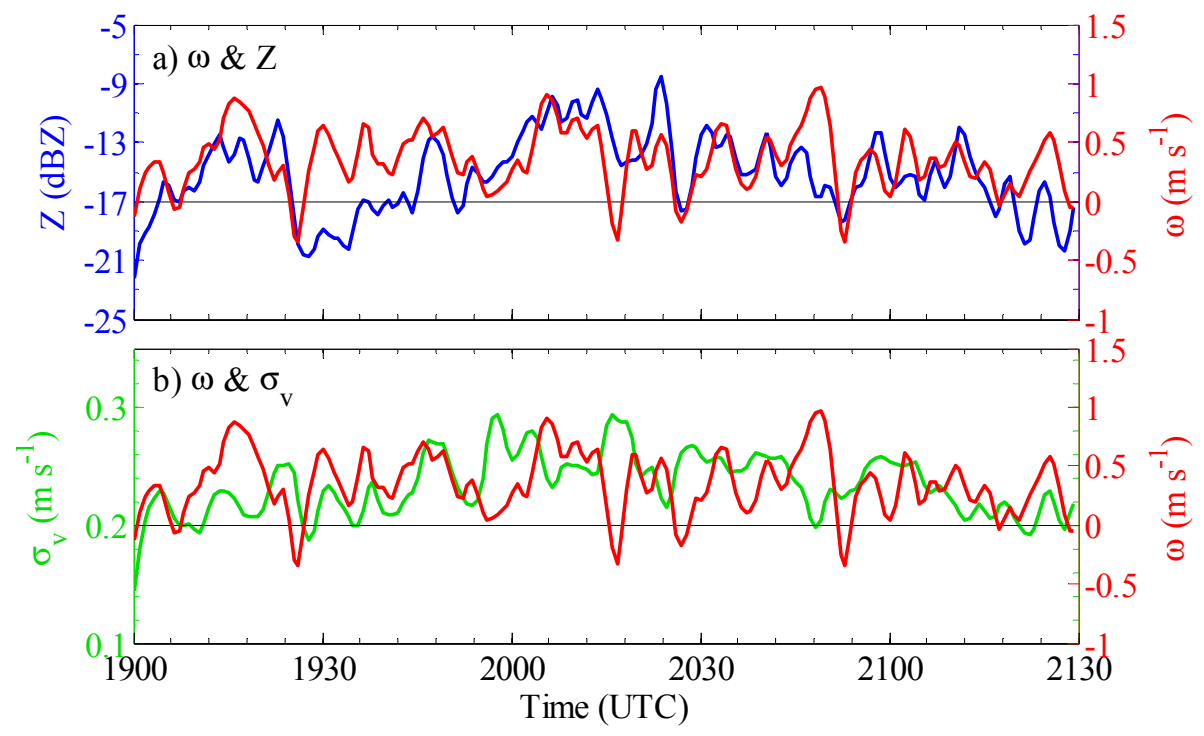

Figure 7. Height-average curves of the reflectivity (blue curve), spectrum width (green curve), and vertical air velocity. 
The second case is a light convective precipitation process collected from 13:40 to 19:00 UTC on August 18, 2015, under the synoptic condition of a plateau shear line, which can be found clearly over the $500 \mathrm{hPa}$ weather chart. The $5.2 \mathrm{~h}$ radar measurements and retrievals of cloud and precipitation are shown in Figure 8. The reflectivity mapping illustrates that there are seven different convective clouds (marked as A, B, C, D, E, F, and G) that pass over the radar site during the observation period. Of these, $\mathrm{C}$ is a layer of stratocumulus located at 7 to 10.5 $\mathrm{km}$ with a reflectivity range from -5 to $15 \mathrm{dBZ}$, while the others are precipitating cumulonimbus clouds with maximum reflectivities of over $25 \mathrm{dBZ}$. A and B are stronger and deeper than D, E, and F, with top heights near $4.5 \mathrm{~km}$ and $7 \mathrm{~km}$, respectively. D, E, and F have 3-4 $\mathrm{km}$ cloud tops. The derived vertical air velocities indicate that the updrafts appear in the upper half of the convections, with strong cores corresponding to positive mean Doppler velocities. The air velocities gradually accelerate from cloud middle to top and may be intimately related to the development level of the cumulonimbus that stronger updrafts are relevant to deeper cloud body. The lower halves of the stratocumulus and cumulonimbus clouds are both dominated by downdrafts or few weak updrafts. As a result, a melting layer near $1.3 \mathrm{~km}$ can be found with sudden increases of mean Doppler velocities and spectrum widths. Figure 9 presents $1.5-6 \mathrm{~km}$ average curves of reflectivity, air vertical velocity, spectrum widths, and raindrop size distribution on the ground collected by a disdrometer. It suggests that the air velocities follow similar trends to the cloud reflectivities and spectrum widths. The updrafts promote the formation of large ice crystals in the interior of the cloud to induce the increases in reflectivities and wider Doppler spectra. This may be different from case one, in which the higher spectrum widths are mostly caused by inhomogeneous and small-scale variations of the vertical air motions. The raindrop size distribution also exhibits a high correlation with the vertical air motions that larger raindrops appear in response to updrafts after a few minutes of delay, such as in cloud B, where the three bursts of larger raindrops are related to the three gusts of updrafts. The intensities of the smallest traced targets imply that the retrievals above $2.01 \mathrm{~km}$ from the CI mode may be relatively accurate with a from -48 to $-30 \mathrm{dBZ}$, while the results below $2.01 \mathrm{~km}$ derived from the PR or BL modes will suffer from a larger bias. Thus, the amendment and merging of our retrieval technique are both necessary and beneficial. 


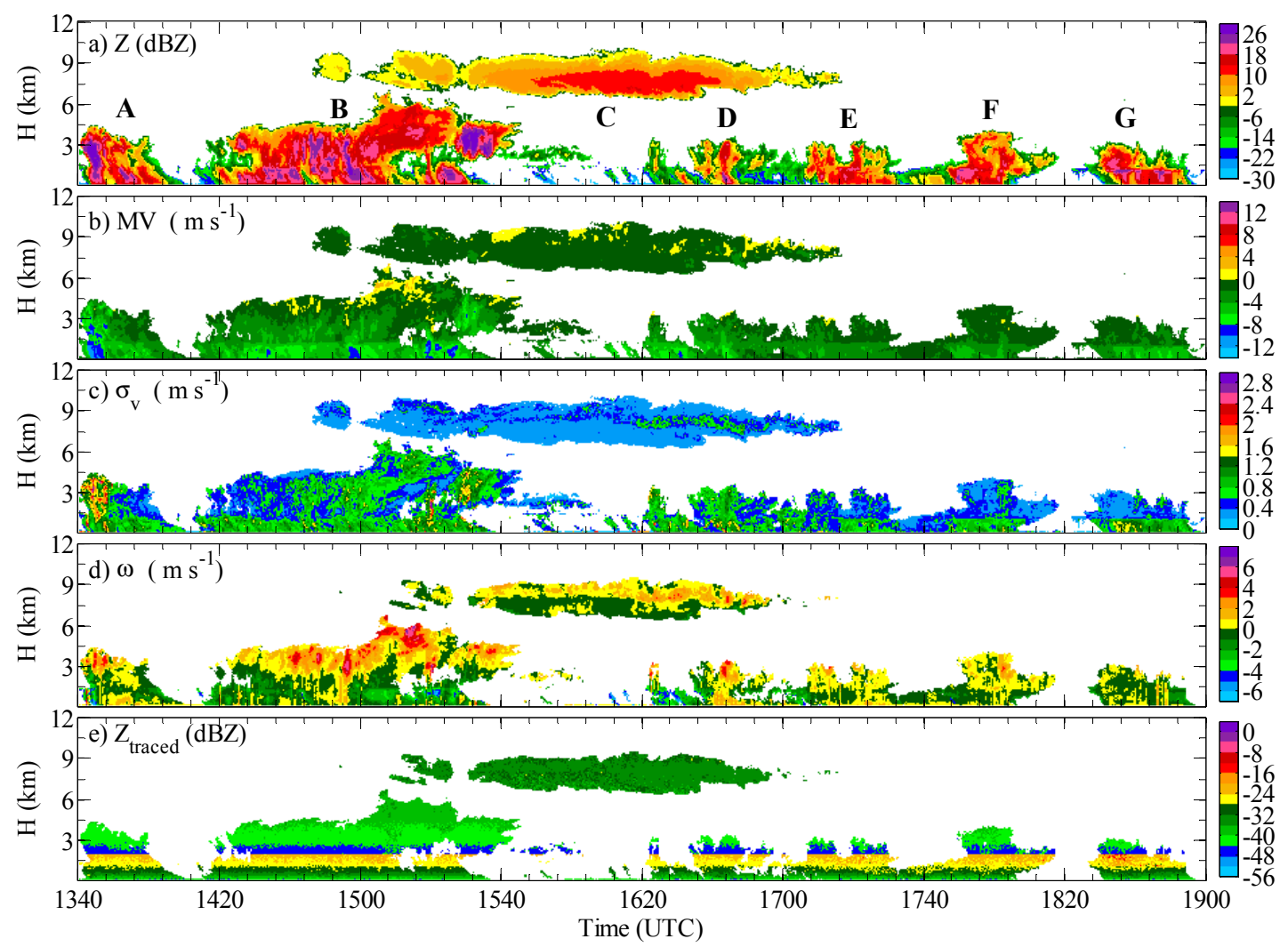

Figure 8. Cloud radar measurements and retrieval results of a light convective precipitation process collected from 13:40 to 19:00 UTC on 18 August 2015.
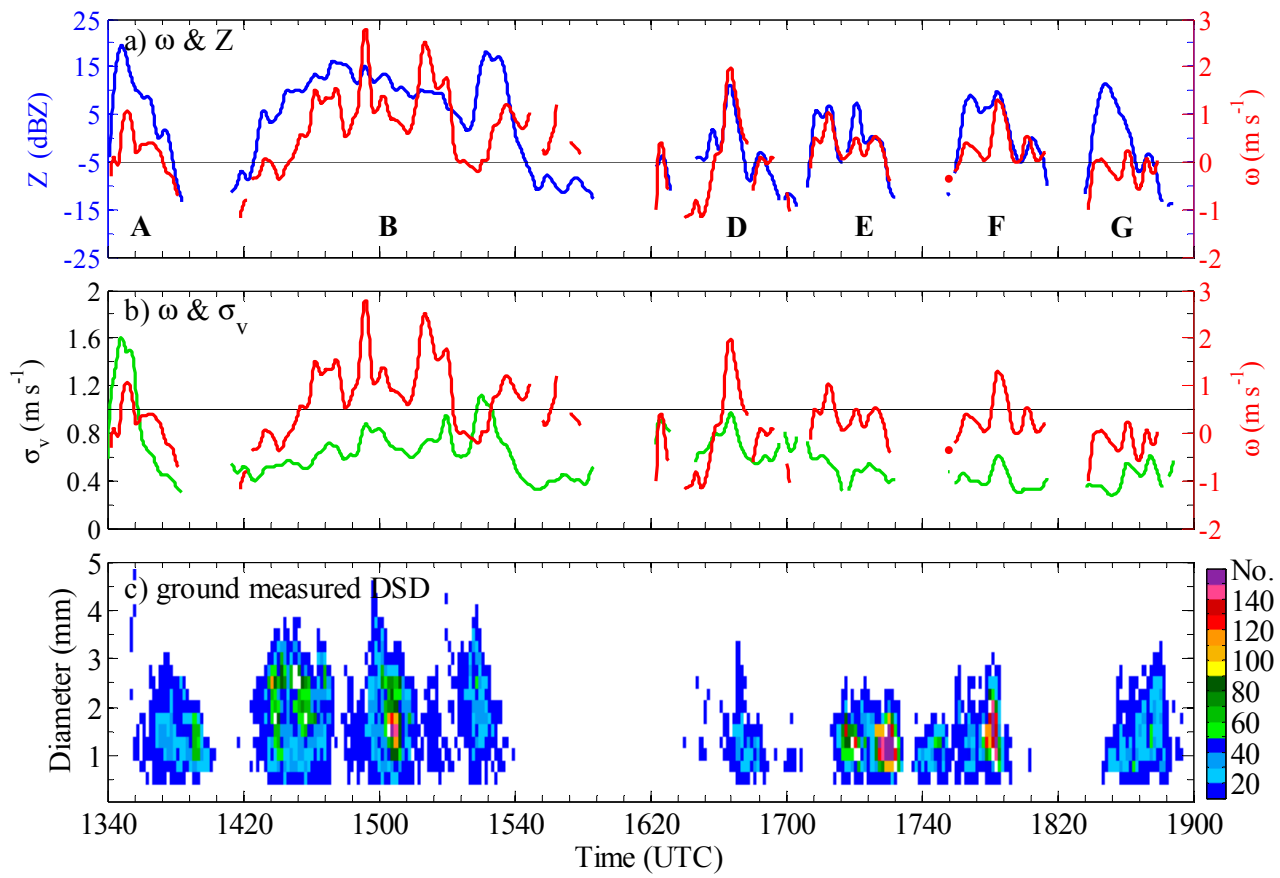

Figure 9. 1.5-6 km average curves of reflectivity, air vertical velocity, spectrum widths and the raindrop size distribution on the ground 


\subsection{Preliminary Validation}

Direct measurements of vertical air motions in convective cloud over TP are still lacking. So, to preliminarily validate the reliability of radar-derived vertical air velocities, indirect retrieval results from a ground-based optical disdrometer were used for comparison.

The disdrometer can simultaneously detect the equivalent diameter $(D)$, concentration $(N)$, and mean vertical velocity $(\bar{V})$ of raindrops near the ground (1.4 $\mathrm{m}$ above the surface) with a 1 min temporal resolution. With the knowledge of raindrop terminal speed $\left(V_{T}\right)$, the vertical air velocity $(\omega)$ can be simply inferred by subtracting $V_{T}$ from $\bar{V}$. For liquid raindrops, many previous studies have indicated that a relationship between $V_{T}$ and $D$ can be established using an exponential function [36-37]:

$$
V_{T}=(9.65-10.3 \exp (-0.6 D)) / f,
$$

where $f$ is a correction factor related to the atmospheric density. Figure 10 shows the frequency distribution of $D$ and $\bar{V}$ of the observed raindrops, collected from July 15 to August 30, 2015, under weak precipitating conditions $\left(10 \leq Z_{e} \leq 25 \mathrm{dBZ}\right)$. A fitting equation with a correction factor of 0.7338 can represent the $V_{T}-D$ relationship of the raindrop on the surface of TP. In the mapping, the measured $\bar{V}$ is marked by a color block, and the deviation between $\bar{V}$ and $V_{T}$ is induced by $\omega$. From the above, the vertical air velocities derived from the disdrometer can be used for comparison with the radar-derived results. Figure 11 shows the time series of retrievals from two instruments for case two, where the radar retrievals are selected from the first detectable ranging gate $(0.15 \mathrm{~km}$ above the surface). The comparison illustrates that the trends of the two retrievals are similar, with the appearances of the updrafts or downdrafts coinciding, but the radar may have a larger fluctuation due to the high temporal resolution. The mean bias of the two retrievals is around $0.5 \mathrm{~m} \mathrm{~s}^{-1}$, but the different heights for the two retrievals and the uncertainty in the disdrometer-derived results could affect the validation.

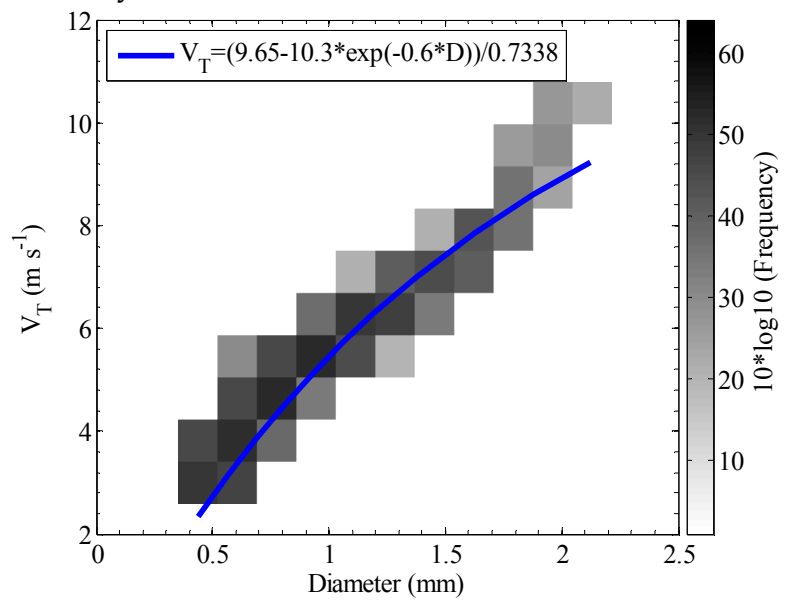

Figure 10. Frequency distribution of $D$ and $\bar{V}$ of the observed raindrops collected from 15 July to 30 August 2015 under weak precipitating conditions, the blue solid line represent a fitting equation for $\mathrm{V}_{\mathrm{T}}-\mathrm{D}$ relationship. 


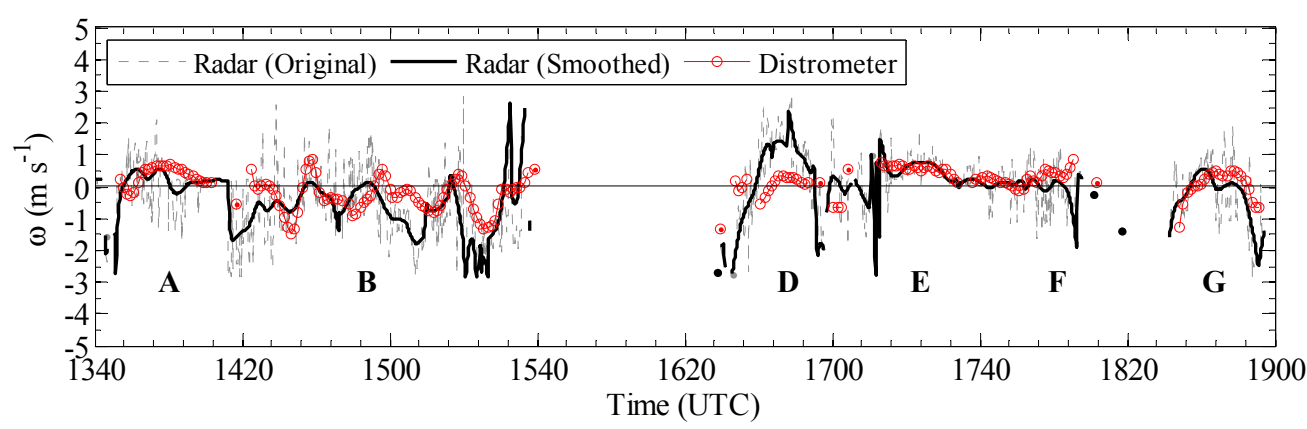

Figure 11. The time series of vertical air velocities derived by radar and disdrometer near the ground for case two, the radar retrievals are selected from the first detectable range gate at $0.15 \mathrm{~km}$ above the surface.

\section{Summary and Conclusions}

Due to large dynamic and thermal effects, convections frequently occur on the TP in the summertime. Measurements of vertical air motions are useful for advancing our understanding of the dynamic and microphysical mechanisms of clouds and can be used to improve the parameterization of current numerical models at many scales. In this paper, a technique was presented for retrieving the high-resolution vertical air velocity in convective cloud over TP by using a vertically pointing Ka-band cloud radar. This technique further develops the smallparticle-traced method and is based on the use of recorded Doppler spectra from a radar and its necessary data processing, over three different modes. Spectral broadening corrections, uncertainty estimations, and result merging were used to ensure more accurate results.

Qualitative analysis of two typical convective cases shows that the retrievals of vertical air velocities are reasonable, meanwhile, some observational features are also found. For the smallscale shallow cumuli, air velocities alternately change across the cumuli, updrafts appear in the middle of cumulous cells, and downdrafts are located on both sides. For precipitating cumulonimbus clouds, updrafts mostly appear in the upper half of the convections and gradually accelerate from cloud middle to cloud top, whereas the lower half of the cumulonimbus is dominated by downdrafts. Vertical air velocity can be intimately related to the development level of the cumulonimbus, with the stronger updrafts corresponding to deeper cloud body. Quantitative retrieval of vertical air motions from a ground-based optical disdrometer was used to preliminarily validate the radar-derived results. The comparison illustrates that the trends of the two retrievals are similar, with the updraft or downdraft appearances coinciding. Cloud radar has much higher resolution retrieval results and can reveal the small-scale variation of vertical air motions. However, we note that the different heights of the two retrievals and the uncertainty in the disdrometer-derived results likely impact the comparison.

Acknowledgement: This study was funded by the National Sciences Foundations of China (The grant numbers are 91337103, 91537214, and 41605022).

Author Contributions: Jiafeng Zheng conceived, designed, processed, analyzed and wrote the manuscript; Liping Liu and Keyun Zhu conducted literature review; Jingya Wu and Binyun Wang processed the data used in this manuscript.

Conflicts of Interest: The authors declare no conflict of interest. 


\section{References}

1. Flohn H., 1968: Contributions to a meteorology of the Tibetan highland. Atmospheric Science Paper. Fort Collins: Colorado State University.

2. Ye Duzheng, Gao Youxi, 1979: Tibetan Plateau Meteorology. Beijing. Science Press, 74-79. (in Chinese)

3. Zhu Guofu, Chen Shoujun, 1999: Convective activities over the Qinghai-Xizang Plateau and adjacent regions in summer of 1995. Plateau Meteorology, 18(1), 9-19. (in Chinese)

4. Xu Xiangde, Zhao Tianliang, Lu Chungu, and Shi Xiaohui, 2014: Characteristics of the water cycle in the atmosphere over the Tibetan Plateau. Acta Meteorologica Sinica, 72(6),1079-1095. (in Chinese)

5. Wu Guoxiong, He Bian, Liu Qimin, Bao Qing, and Ren Rongcai, 2016: Recent progressed on dynamics of the Tibetan Plateau and Asian summer monsoon. Chinese Journal of Atmospheric Sciences, 40(1), 22-32. (in Chinese)

6. Qian Zhengan, Wu Tongwen, Lv Shihua, and Jiao Yanjun, 1998: Numerical simulation of northwest China arid climate formation-effects of the Qinghai-Xizang plateau terrain and circulation field. Chinese Journal of Atmospheric Sciences, 22(5), 753-762. (in Chinese)

7. Jiang Jixi and Fan Meizhu, 2002: Convective clouds and mesoscale convective systems over the Tibetan Plateau in summer. Chinese Journal of Atmospheric Sciences, 26(2), 263-270. (in Chinese)

8. Tao, S. Y., and Ding, Y. H., 1981: Observational evidence of the influence of the Qinghai-Xizang(Tibet) plateau on the occurrence of heavy rain and severe convective storms in China. Bull. Amer. Meteor. Soc., 62, 23-30.

9. Xu Xiangde, Zhou Mingxu, and Chen Jiayi, 2001: Thermal and dynamic integrated physical images of land-atmosphere over the Tibetan Plateau. Science in China (Series D), 31(5), 428-440. (in Chinese)

10. Liu L. P., Zheng J. F., Ruan Z., and et al., 2015: Comprehensive radar observations of clouds and precipitation over the Tibetan Plateau and Preliminary analysis of cloud properties. J. Meteor. Res., 29(4), 546-561.

11. Wang C. H., Shi. H. X., Hu H. L., Wang Y., and Xi B., 2015: Properties of cloud and precipitation over the Tibetan Plateau. Adv. Atmos. Sci., 32(11), 1504-1516.

12. Lhermitte, R., 1990: Attenuation and scattering of millimeter wavelength radiation by clouds and precipitation. J. Atmos. Oceanic Technol., 7, 464-479.

13. Clothiaux,E. E., M.A. Miller, B.A. Albrecht, T. P. Ackerman, J. Verlinde, D. M. Babb, R. Peters, and W. J. Syrett, 1995: An evaluation of a 94-GHz radar for remote sensing of cloud properties. J. Atmos. Oceanic Technol., 12, 201-229.

14. Kollias, P., E. Clothiaux, M. Miller, B. Albrecht, and G. T. Ackerman, 2007: Millimeter-wavelength radars: New frontier in atmospheric cloud and precipitation research. Bull. Amer. Meteor. Soc., 88, 1608-1624.

15. Illingworth, A. J., and Coauthors, 2007: Cloudnet. Bull. Amer. Meteor. Soc.,88, 883-898.

16. Ulrich Görsdorf and Volker Lehmann, Matthias Bauer-Pfundstein, Gerhard Peters, Dmytro Vavriv, Vladimir Vinogradov, and Vadim Volkov, 2015: A 35-GHz polarimetric Doppler radar for long-term observations of cloud parameters-description of system and data processing. J. Atmos. Oceanic Technol., 32, 675-690.

17. Gossard, E. E. and R. G. Strauch, 1983: Radar observations of clear air and clouds. Elsevier, 280 pp.

18. Kollias, P., B. A. Albrecht, and F. D. Marks Jr., 2003: Cloud radar observations of vertical drafts and microphysics in convective rain. J. Geophys. Res., 108, 40-53.

19. Rogers R R., 1964: An extension of the Z-R relation for Doppler radars. The 11th Weather Radar Conference AMS, Boston, MA., Goulder, Co., Sept., 14-18.

20. Sekhon, R. S., and R. C. Srivastava, 1971: Doppler radar observations of drop-size distribution in a thunderstorm. J. Atmos. Sci., 28: 983-994.

21. Hauser D., Amayenc P., 1981: A new method for deducing hydrometeor-size distributions and vertical air motions from Doppler radar measurements at vertical incidence. J. Appl. Meteor., 20, 547555. 
22. Giangrande S. E., Collis S., and Straka J., 2013: A summary of convective-core vertical velocity properties using ARM UHF wind profilers in Oklahoma. J. Appl. Meteor. Climatol., 52(10), 22782295.

23. Lhermitte, R., 1988: Observations of rain at vertical incidence with a $94 \mathrm{GHz}$ Doppler radar: An insight of Mie scattering. Geophys. Res. Lett., 15, 1125 - 1128.

24. Gossard E. E., 1994: Measurement of cloud droplet size spectra by Doppler radar. J. Atmos. Oceanic Technol., 11,712-726.

25. Kollias, P., B. A. Albrecht, R. Lhermitte, and A. Savtchenko, 2001: Radar observations of updrafts, downdrafts, and turbulence in fair weather cumuli, J. Atmos. Sci., 58, 1750-1766.

26. Shupe, M. D., P. Kollias, M. Poellot, and E. Eloranta, 2004: Deriving mixed-phase cloud properties from Doppler radar spectra. J. Atmos. Oceanic. Technol., 65, 1304-1322.

27. Shupe, M. D., P. Koliias,S. Y. Matrosov, and T. L. Schneider, 2008: On deriving vertical air motions from cloud radar Doppler spectra. J. Atmos. Oceanic. Technol., 25,547-557.

28. Liu, L. P., J. F. Zheng, and J. Y. Wu, 2017: A Ka-band solid-state transmitter cloud radar and data merging algorithm for its measurements. Adv. Atmos. Sci., 34(4), 545-558.

29. Zheng Jiafeng, Liu Liping, Zeng Zhengmao, Xie Xiaolin, Wu Jingya, and Feng Kai, 2016: Ka-band millimeter wave cloud radar data quality control. J. Infrared Millim. Waves, 35(6), 748-757.

30. Hildebrand, P. H., and R. S. Sekhon, 1974: Objective determination of the noise level in Doppler spectra. J. Appl. Meteor., 13, 808-811.

31. Luke E. P. and P. Kollias, 2013: Separating cloud and drizzle radar moments during precipitation onset using Doppler spectra. J. Atmos. Oceanic Technol., 30, 1656-1671.

32. Monique P., A. Sy, A. Garrouste, and J. Delcourt, 1997: Statistical characteristics of the noise power spectral density in UHF and VHF wind profilers. Radio Scienc, 32(3): 1229-1247.

33. Bouniol, D., A. J. Illingworth, and R. J. Hogan, 2003: Deriving turbulent kinetic energy dissipation rate within clouds using ground based $94 \mathrm{GHz}$ radar. Preprints, 31st Conf. on Radar Meteorology, Seattle, WA, Amer. Meteor. Soc., 193-196.

34. O'Connor, E. J., R. J. Hogan, and A. J. Illingworth, 2005: Retrieving stratocumulus drizzle parameters using Doppler radar and lidar. J. Appl. Meteor., 44, 14-27.

35. Warner, J., 1977: Time variation of updraft and water content in small cumulus clouds. J. Atmos. Sci., 34, 1306-1312.

36. Gunn R, Kinzer G D., 1949: The terminal velocity of fall for water droplets in stagnant air. Journal of Meteorology, 6(4), 243-248.

37. Foote G B, Du Toit, P. S., 1969: Terminal velocity of raindrops aloft. Journal of Applied Meteorology, 8(2), 249-253. 